Agronomie

\title{
Effet d'un déficit hydrique sur le trèfle blanc (Trifolium repens L) I. Rôle d'un apport de potassium
}

\author{
L Shamsun-Noor*, C Robin , A Guckert \\ INRA, Ecole Nationale Supérieure d'Agronomie et des Industries Alimentaires, laboratoire de phytotechnie, \\ 2, avenue de la Forêt de Haye 54500 Vandceuvre, France
}

(Reçu le 18 août 1988; accepté le 12 octobre 1989)

\begin{abstract}
Résumé - Le comportement du trèfle blanc (Trifolium repens L cv Crau) est étudié en situation de contrainte hydrique et après réhydratation en liaison avec la fertilisation potassique. Le déficit en eau occasionne une décroissance progressive importante du potentiel hydrique des feuilles et une fermeture rapide des stomates. Ces manifestations sont accompagnées d'une chute de l'activité photosynthétique et de la fixation symbiotique de l'azote. En présence de potassium, la diminution de l'activité fixatrice est significativement ralentie. L'action favorable du potassium est également observée lors de la réhydratation des plantes soumises au déficit. Le rétablissement du potentiel hydrique et de la fixation symbiotique d'azote est plus important en présence de potassium. La fertilisation potassique exerce un effet positif sur la production de matière sèche aérienne aussi bien dans le cas des plantes soumises au déficit que des plantes régulièrement arrosées.

Les résultats montrent qu'un apport de potassiuin favorise la résistance du trèfle blanc au manque d'eau.
\end{abstract}

trèfle blanc / sécheresse / potassium / potentiel hydrique / stomate / photosynthèse / azote

\begin{abstract}
Summary - Effect of water stress on white clover (Trifolium repens L) I. Role of a $\mathbf{K}$ supply. The effects of successive water stress and irrigation on white clover (Trifolium repens $L$ cv Crau) photosynthetic activity, nitrogen fixation, predawn leaf water potential and stomatal resistance were studied in relation to potassium supply. Water deficit induced a gradual decrease in the predawn leaf water potential and a rapid stomatal closure (figs 1 and 2). Nitrogen fixation and photosynthetic activity were also reduced (fig 4 and table 1). The decrease in nitrogen fixation activity was significantly lower with $K$ supply (fig 4). For the same water potential and the same stomatal resistance, nitrogen fixation activity (ARA's method) was higher in presence of potassium fertilization (fig 5 a, b). The positive effect of $K$ was also observed for the rehydrated plants. Water potential and symbiotic nitrogen fixation recovery was more significant with $K$ supply. Potassium fertilization increased dry matter production in stressed plants as in controls (fig 3). These results showed that $K$ supply increased white clover resistance to water stress.
\end{abstract}

white clover / drought / potassium / water potential / stomata / photosynthesis / nitrogen

\section{INTRODUCTION}

L'alimentation hydrique constitue un facteur essentiel de la productivité. L'activité fixatrice d'azote chez les légumineuses dépend de la disponibilité en eau du sol (Sprent, 1972; Obaton et al, 1982).

L'élément potassium intervient aussi au niveau du statut hydrique de la plante (Mengel, 1984).
De nombreux auteurs ont souligné le rôle important joué par le potassium dans la résistance à la contrainte hydrique (Raschke, 1975; Dhindsa et al, 1975; Zimmerman, 1978; Saxena, 1985).

Cet élément intervient en effet dans les phases primaires de l'assimilation du $\mathrm{CO}_{2}$ par les organes chlorophylliens, par action directe sur les mécanismes osmotiques d'ouverture des stomates (Humble et Raschke, 1971). 
Au-delà de ces acquis relatifs à la régulation stomatique, peu de travaux ont concerné le rôle du potassium sur l'évolution de l'activité nitrogénase au cours d'un déficit hydrique chez les légumineuses pérennes. Guckert et Laperrière (1987) ont mis en évidence un effet favorable de cet élément sur le potentiel hydrique et sur l'activité fixatrice du trèfle blanc en situation de déficit en eau.

La présente étude vise à approfondir ces premiers résultats et à déterminer l'effet combiné des facteurs : alimentation hydrique et fertilisation potassique.

Dans ce but, on a cultivé le trèfle blanc sur un sol déficient en K (amendé ou non en cet élément) et comparé différentes fonctions physiologiques, en situation de déficit hydrique:

- potentiel hydrique de base et résistance stomatique;

- photosynthèse;

- fixation symbiotique de l'azote.

L'évolution des biomasses aériennes a également été observée. Le rétablissement ou le degré d'altération de ces fonctions a ensuite été évalué après une réalimentation en eau des plantes ayant subi la phase de dessèchement.

\section{MATÉRIEL ET MÉTHODES}

\section{Conditions de croissance}

L'ensemble de l'expérimentation s'est déroulée en vases de végétation, en conditions contrôlées. Le cultivar de trèfle blanc étudié est Crau; il appartient au type Ladino. Ce choix s'appuie sur les résultats antérieurs (Shamsun-Noor et al, 1989) d'un criblage de cultivars pour leurs performances en condition de déficit hydrique. Crau est apparu comme le cultivar le plus tolérant parmi les 4 testés.

Les plantes sont semées en pots de $4 \mathrm{~kg}$; leur densité est progressivement ramenée à 15 plantules par pot. Le sol, fourni par le centre de recherches d'Aspach (Société commerciale des potasses et de l'azote) a été prélevé à Weisslingen dans le nord de l'Alsace. II s'agit d'un sol sur grès vosgien dont la particularité intéressante réside dans sa faible teneur en potassium (16,8\% d'argiles, $32,3 \%$ de limons fins, $29 \%$ de limons grossiers, $19,1 \%$ de sable, potassium $\left(\mathrm{K}_{2} \mathrm{O}\right)=0,012 \%$, $\mathrm{pH}=6,2$, capacité au champ $=36,6 \%$ d'humidité pondérale).

Les pots de trèfle sont répartis en 2 lots:

- lot $K_{0}$ correspondant au sol sans apport de K;

- lot $K_{1}$ sol ayant reçu avant le semis l'équivalent de $300 \mathrm{U}$ de $\mathrm{K}_{2} \mathrm{O}$ sous forme de $\mathrm{K}_{2} \mathrm{SO}_{4}$.

Pendant toute la période de croissance précédant les mesures, la culture est conduite en serre. L'humidité du sol est maintenue à $70 \%$ de la capacité au champ (soit $25,6 \%$ d'humidité pondérale), niveau compatible avec une activité maximale de la fixation symbiotique de l'azote (Laperrière, 1984) sur ce type de substrat limono-sableux. Une semaine avant l'expé- rience, les 2 lots sont transférés en chambre phytotronique [photopériode de $14 \mathrm{~h}$, thermopériode $21^{\circ} \mathrm{C}$ le jour $/ 18{ }^{\circ} \mathrm{C}$ la nuit, intensité lumineuse voisine de $300 \mu \mathrm{mol}$ (photons) $\cdot \mathrm{m}^{-2} \cdot \mathrm{s}^{-1}$, humidité relative $\left.76 \%\right]$. Le déficit est établi par suppression de l'arrosage quotidien. Chaque lot $\left(K_{0}\right.$ et $\left.K_{1}\right)$ présente donc 2 traitements :

- témoin (maintenu à $70 \%$ de la $\mathrm{CC}$ ) $=\mathrm{K}_{0 \mathrm{Tém}}, \mathrm{K}_{1 \mathrm{Tém}}$;

- déficit (par arrêt de l'arrosage) $=K_{0 \text { Déf }}, K_{1 \text { Dét }}$.

\section{Méthodes}

La plupart des méthodes utilisées ont été décrites précédemment (Shamsun-Noor et al, 1989).

\section{Mesure du potentiel hydrique des feuilles}

L'état interne de l'eau libre d'une plante est apprécié par la mesure du potentiel hydrique de sève. Cette valeur est obtenue à l'aide de la méthode de la chambre à pression de Schölander (Schölander et al, 1965). Il est ainsi possible de déterminer le potentiel hydrique de base, qui correspond au potentiel mesuré en fin de nuit lorsque la demande climatique est nulle (Aussenac et Granier, 1978).

La première mesure de potentiel hydrique a été effectuée le jour de l'arrêt de l'arrosage. Chaque mesure a fait l'objet de 8 répétitions.

\section{Mesure de la résistance stomatique}

La mesure de la résistance stomatique est obtenue par l'utilisation du poromètre de Van Bavel et al (1965) dont le principe est de déterminer les variations d'humidité relative au niveau d'une atmosphère en contact avec les cellules foliaires et les stomates. Une sonde hygrométrique résistive donne directement l'humidité relative de la chambre de mesure. Chaque mesure a fait l'objet de 4 répétitions.

\section{Mesure de l'activité photosynthétique}

La technique utilisée, préalablement décrite (Shamsun-Noor et al, 1989), consiste à déterminer l'assimilation nette d'un couvert de trèfle par un suivi de l'évolution de la teneur en $\mathrm{CO}_{2}$ dans l'enceinte de mesure.

Les résultats d'assimilation nette sont donnés en volume par million (vpm) de $\mathrm{CO}_{2}$ assimilé par h et par pot. Les valeurs moyennes sont issues de 3 répétitions.

\section{Mesure de l'activité fixatrice d'azote}

La fixation symbiotique de l'azote atmosphérique est couramment appréhendée par la méthode indirecte de mesure de l'activité réductrice d'acétylène de la nitrogénase, ou méthode ARA (Koch et Evans, 1966).

L'ARA est exprimée en moles d'éthylène produites par heure d'incubation et par pot (méthode non destructive). Chaque mesure a fait l'objet de 4 répétitions. 


\section{Analyse statistique}

L'analyse statistique de l'ensemble des données repose sur des analyses de variance et sur des comparaisons de moyennes selon le test de Newman-Keuls: dans les tableaux de résultats, les moyennes suivies de la même lettre ne sont pas significativement différentes au seuil de $5 \%$. Les segments verticaux portés sur les figures représentent les écarts types par rapport à la moyenne.

\section{RÉSULTATS}

\section{Potentiel hydrique de base}

L'apport de $K$ ne modifie pas le potentiel hydrique de base des plantes normalement pourvues en eau (plantes témoins). En revanche, le déficit hydrique exerce un effet hautement significatif $(P<0,001)$ sur le potentiel de base dès le $2^{\theta}$ j qui suit l'arrêt de l'alimentation en eau. La chute du potentiel de base est sévère à partir du $4^{\theta} \mathrm{j}$, avec cependant un meilleur comportement du lot $K_{1}$ ayant reçu un apport initial en potassium (fig 1).

Le potentiel de base des plantes $K_{1}$ est revenu au niveau des témoins $5 \mathrm{j}$ après la réhumidification du sol. Les plantes soumises à la contrainte $\left(K_{0}\right)$ n'atteignent le niveau de leur témoin que le dernier jour. On assiste à l'annulation progressive de l'effet $K$ en fin d'expérience $\left(j_{12} a j_{15}\right)$.

L'aspect morphologique des deux séries de plantes est très différent après $8 \mathrm{j}$ de déficit, les feuilles du lot $K_{0}$ se dessèchent alors que celles du lot $K_{1}$, quoique faiblement turgescentes, sont encore vertes. Après rétablissement de l'arrosage, ces dernières reprennent rapidement leur port initial. Pour le lot $K_{0}$, de nouvelles feuilles sont initiées en remplacement des feuilles fanées et nécrosées.

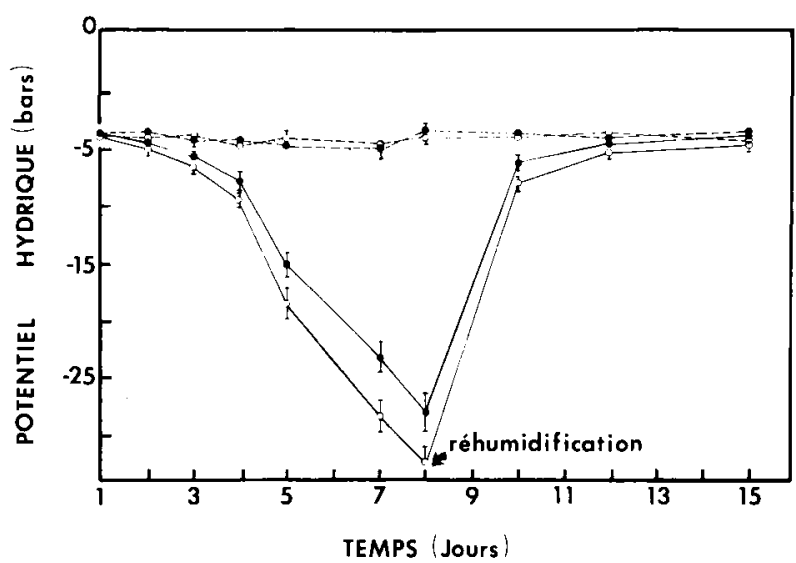

Fig. 1. Evolution du potentiel hydrique de base (bars) des plantes de trèfle blanc (Crau) subissant $(-$ ) ou non (- -) un déficit hydrique à 2 niveaux de potassium $\left(\mathrm{K}_{0}\right.$ : $\mathrm{O}$ et $\mathrm{K}_{1}$ : - équivalent à $300 \cup \mathrm{K}_{2} \mathrm{O}$ ).

\section{Activité photosynthétique nette et résistance stomatique}

L'effet du déficit hydrique sur la photosynthèse est également significatif à partir du $3^{\ominus}$ j (tableau I). L'effet du potassium se traduit sur les plantes ayant subi le déficit par une activité photosynthétique plus élevée même si les différences ne sont pas significatives à chaque observation. Après réhumidification, l'activité photosynthétique nette n'est pas totalement restaurée et ne rejoint pas la valeur de celle des témoins, notamment pour le lot $\mathrm{K}_{0}$; ce phénomène peut être mis en relation avec l'altération de l'appareil photosynthétique, consécutive au déficit hydrique.

Les valeurs mesurées de la résistance stomatique (RS) des plantes témoins restent faibles $\left(0,8-2,8 \mathrm{~s} \cdot \mathrm{cm}^{-1}\right)$ et stables pendant l'expérience (fig 2).

En revanche, les plantes en déficit présentent des différences considérables par rapport aux témoins; le déficit en eau entraîne un accroissement rapide de la $R S$ (supérieur à $200 \mathrm{~s}^{-1} \cdot \mathrm{cm}^{-1}$ ). $L^{\prime}$ 'écart entre les deux courbes $K_{0}$ et $K_{1}$ est faible (non significatif au seuil de $5 \%$ ).

Après réhumidification, la résistance stomatique chute très rapidement; les valeurs rejoignent celles des témoins arrosés plus rapidement que pour le potentiel hydrique. En $48 \mathrm{~h}$, la RS passe de $200-220 \mathrm{~s} \cdot \mathrm{cm}^{-1}$ à $2 \mathrm{~s} \cdot \mathrm{cm}^{-1}$.

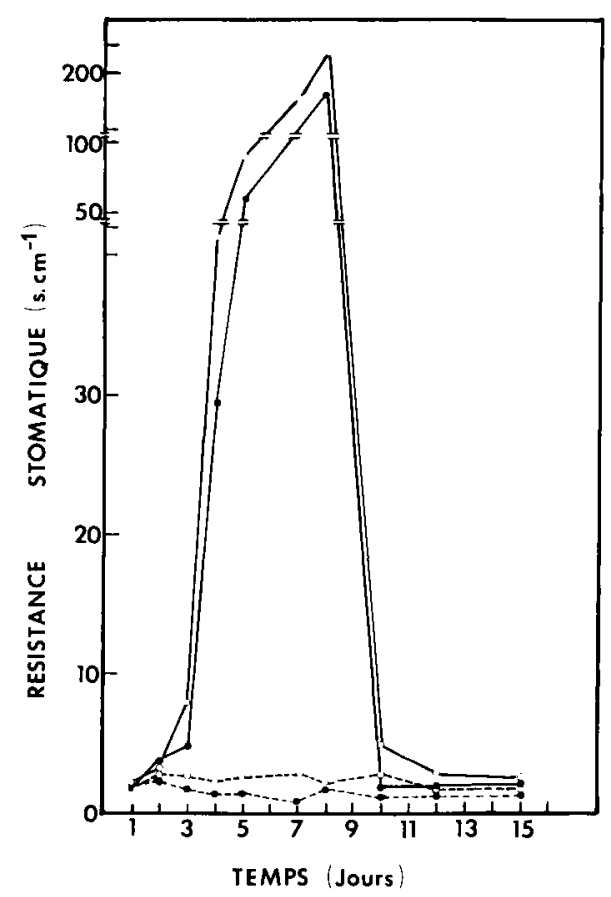

Fig. 2. Evolution de la résistance stomatique $\left(\mathrm{s} \cdot \mathrm{cm}^{-1}\right)$ des plantes de trèfle blanc (Crau) subissant $(-$ ou non $(--)$ un déficit hydrique à 2 niveaux de potassium $\left(\mathrm{K}_{0}: \mathrm{O}\right.$ et $\mathrm{K}_{1}$ : - équivalent à $300 \cup \mathrm{K}_{2} \mathrm{O}$ ). 
Tableau I. Analyse de variance des mesures d'activité photosynthétique nette $\left(\mathrm{mg} \mathrm{CO}_{2} \cdot \mathrm{h}^{-1} \cdot \mathrm{pot}^{-1}\right)$ du cultivar Crau. NS : non significatif au seuil de $5 \%, S$ : significatif à $5 \%\left(^{\star}\right), 1 \%\left(^{\star \star}\right), 0,1 \%\left(^{\star \star \star}\right)$.

\begin{tabular}{|c|c|c|c|c|c|c|c|c|c|c|}
\hline Temps & 1 & 2 & 3 & 4 & 5 & 7 & 8 & 10 & 12 & 15 \\
\hline $\begin{array}{l}\text { Effet du facteur } \\
\text { "déficit hydrique" }\end{array}$ & NS & NS & $S^{* * \star}$ & $S^{\star \star}$ & $S^{\star \star \star}$ & $S^{\star \star \star}$ & $S^{\star \star \star *}$ & $S^{* * *}$ & $S^{*}$ & $S^{*}$ \\
\hline $\begin{array}{l}\text { Témoin (Tém) } \\
\text { Déficit (Déf) }\end{array}$ & $\begin{array}{l}45,86 a \\
44,56 a\end{array}$ & $\begin{array}{l}43,39 a \\
40,40 a\end{array}$ & $\begin{array}{l}44,78 a \\
34,93 b\end{array}$ & $\begin{array}{l}44,57 a \\
31,00 b\end{array}$ & $\begin{array}{l}44,46 a \\
23,90 b\end{array}$ & $\begin{array}{r}45,26 a \\
8,73 b\end{array}$ & $\begin{array}{r}44,07 a \\
3,97 b\end{array}$ & $\begin{array}{l}45,24 a \\
23,08 b\end{array}$ & $\begin{array}{l}45,33 a \\
32,43 b\end{array}$ & $\begin{array}{l}45,44 a \\
38,15 b\end{array}$ \\
\hline $\begin{array}{l}\text { Interaction } \\
\text { Déficit } \\
\text { Potassium }\end{array}$ & NS & NS & NS & NS & NS & NS & NS & NS & NS & NS \\
\hline $\begin{array}{l}\text { Tém - } K_{0} \\
\text { Tém }-K_{1} \\
\text { Déf- } K_{0} \\
\text { Déf - } K_{1}\end{array}$ & $\begin{array}{l}45,60 a \\
46,13 a \\
43,38 a \\
45,74 a\end{array}$ & $\begin{array}{l}42,63 a \\
44,15 a \\
39,66 a \\
41,14 a\end{array}$ & $\begin{array}{l}43,70 a \\
45,87 a \\
34,04 b \\
35,83 b\end{array}$ & $\begin{array}{l}44,89 a \\
44,25 a \\
29,78 b \\
32,23 b\end{array}$ & $\begin{array}{l}43,80 a \\
45,12 a \\
21,34 b \\
26,46 b\end{array}$ & $\begin{array}{c}45,59 a \\
44,92 a \\
7,06 b \\
10,41 b\end{array}$ & $\begin{array}{r}43,06 a \\
45,08 a \\
2,36 b \\
5,59 b\end{array}$ & $\begin{array}{l}45,86 a \\
44,61 a \\
19,89 b \\
26,26 b\end{array}$ & $\begin{array}{l}44,49 a \\
46,17 a \\
29,44 b \\
35,42 b\end{array}$ & $\begin{array}{l}45,18 a \\
45,70 a \\
35,46 b \\
40,84 b\end{array}$ \\
\hline
\end{tabular}

\section{Evolution de la matière sèche (MS) des par- ties aériennes}

Les parties aériennes comprennent les limbes, pétioles et stolons de l'ensemble des plantes d'un seul pot pour chaque modalité. Le manque d'eau induit une réduction importante de l'accumulation de MS dans les parties aériennes des plantes soumises au déficit (fig 3 ) $5 \mathrm{j}$ après la suppression de l'arrosage pour les plantes $\mathrm{K}_{0}$ et $\mathrm{K}_{1}$.

Nous n'avons pas observé d'effet de la réhydratation sur l'évolution de la biomasse.

La MS produite discrimine très nettement les plantes avec ou sans fertilisation $\mathrm{K}$, aussi bien dans le cas des plantes soumises au déficit que dans celui des plantes régulièrement arrosées; l'apport de potassium entraîne systématiquement une augmentation de la biomasse aérienne.

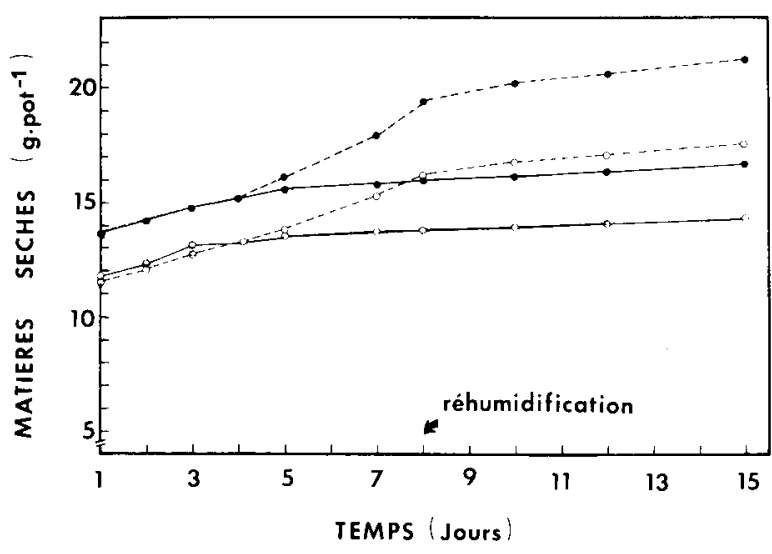

Fig. 3. Evolution de la masse de matière sèche des parties aériennes $(\mathrm{g} / \mathrm{pot})$ de Crau subissant $(-)$ ou non $(--)$ un déficit hydrique à 2 niveaux de potassium $\left(\mathrm{K}_{0}: \mathrm{O}\right.$ et $\mathrm{K}_{1}$ : - = équivalent à $300 \cup \mathrm{K}_{2} \mathrm{O}$ )
En fin d'expérience, les écarts de rendement en MS entre les plantes $K_{0}$ et $K_{1}$ est de $17,5 \%$ pour les témoins et $14,2 \%$ pour les plantes ayant subi le déficit.

\section{Activité réductrice d'acétylène de la nitrogé- nase}

L'effet du déficit en eau sur l'ARA est significatif (au risque $1 \%$ ) par rapport aux lots témoins dès le $3^{e} \mathrm{j}$ (fig 4).

Des différences importantes et significatives entre les 2 niveaux de $\mathrm{K}$ apparaissent à partir du $5^{\ominus} \mathrm{j}$. Les plantes bien pourvues en $\mathrm{K}$ conservent, lors du déficit, un niveau d'ARA supérieur aux plantes non fertilisées.

Après réhumidification, on note que les plantes ayant reçu un apport de $\mathrm{K}$ retrouvent un niveau de fixation supérieur à celui des plantes $K_{0}$. En fin d'expérience, l'ARA du lot $K_{1}$ représen-

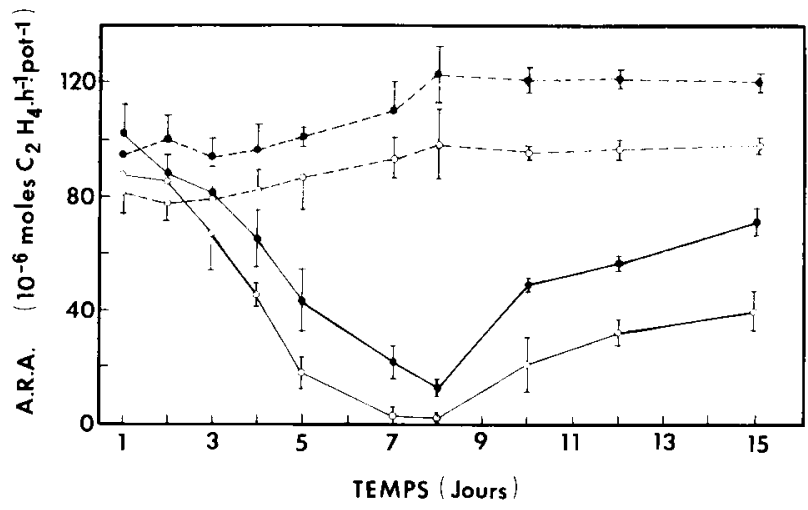

Fig. 4. Evolution de l'activité réductrice d'acétylène $\left(10^{-9} \mathrm{~mol}\right.$ $\mathrm{C}_{2} \mathrm{H}_{4} \cdot \mathrm{h}^{-1} \cdot$ pot) de Crau subissant $(\longrightarrow$ ou non $(-\ldots)$ un déficit hydrique à 2 niveaux de potassium $\left(\mathrm{K}_{0}: \mathrm{O}\right.$ et $\mathrm{K}_{1}: 0$ = équivalent à $300 \cup \mathrm{K}_{2} \mathrm{O}$ ). 
te $60 \%$ de la valeur du témoin alors que le lot $\mathrm{K}_{0}$ n'a récupéré que $40 \%$ de la valeur d'ARA de son témoin.

Contrairement aux autres paramètres mesurés (potentiel hydrique, résistance stomatique) pour lesquels on constate une récupération rapide et un retour aux valeurs des témoins, l'activité fixatrice des plantes soumises à un déficit hydrique puis réalimentées en eau n'est pas entièrement restaurée en fin d'expérience.

\section{DISCUSSION ET CONCLUSIONS}

L'apport de $K$ exerce un effet favorable sur la biomasse aérienne du trèłle blanc. Ce résultat est en accord avec des observations effectuées aussi bien sur des légumineuses pérennes telles que la luzerne (Duke et al, 1980; Barbarick, 1985), que sur des annuelles telles que le haricot (Mengel et Arneke, 1982) et le pois (Wahab, 1984). Dans nos conditions expérimentales, les plantes placées en situation de déficit hydrique mais fertilisées avec $K$ fournissent une production de trèfle équivalente à celle du sol témoin non enrichi en $\mathrm{K}$.

Les plantes en situation de déficit hydrique présentent une augmentation importante de la résistance stomatique. Cependant, cette expérimentation n'a pas permis de montrer une influence significative du potassium dans la régulation stomatique, observée notamment par Brag (1972) sur le blé et le pois.

En revanche, nous avons montré un effet positif de l'apport de K sur l'évolution de l'activité photosynthétique lors du déficit. Peoples et Koch (1979) indiquent pour Medicago sativa un effet favorable du $\mathrm{K}$ sur l'assimilation du $\mathrm{CO}_{2}$. Le potassium tend à augmenter la surface photosynthétique active par une initiation et un développement plus importants des feuilles, avec un taux de fixation du $\mathrm{CO}_{2}$ par unité de surface amélioré (Cooper et al, 1967).

L'effet favorable du $\mathrm{K}$ s'exerce aussi au niveau de la fixation de l'azote; les plantes bien alimentées en $\mathrm{K}$ présentent une activité fixatrice plus élevée que les plantes carencées.

De plus, en situation de manque d'eau, la diminution de l'ARA est de moindre amplitude en présence de potassium. Cette observation confirme les résultats antérieurs de Guckert et Laperrière (1987); l'apport de K permet de maintenir un système fixateur encore efficient malgré un déficit hydrique sévère.

Après réhydratation, l'ARA n'atteint pas la valeur des témoins; l'hypothèse d'un effet direct du dessèchement sur les nodosités peut être avancée, en liaison avec les acquis de la bibliographie (Albrecht et al, 1984).

Collins et Lang (1985) ont montré que l'addition de $\mathrm{K}$ augmentait le taux de fixation chez le trèfle violet et le lotier sans exercer d'effet sur le nombre de nodosités.

Chez la luzerne en cours de repousse, Barta (1982) indique que l'effet favorable de l'apport de $K$ sur le taux de fixation est probablement lié à une augmentation du transport des assimilats vers les nodosités et à une meilleure utilisation des chaînons carbonés pour la synthèse des acides aminés.

Dans le présent travail, il s'avérait intéressant d'établir des corrélations entre l'ARA et le potentiel hydrique de base et entre l'ARA et la résistance stomatique.

Ces relations (établies avec les valeurs moyennes) ne sont pas linéaires : leur ajustement est de type exponentiel. En vue de mettre en évidence l'existence d'une différence entre les traitements, ces courbes ont été linéarisées par une transformation log des valeurs moyennes d'ARA (exprimée en $10^{-6} \mathrm{~mol}\left(\mathrm{C}_{2} \mathrm{H}_{4}\right) \cdot h^{-1} \cdot$ pot $\left.^{-1}\right)$.

La relation entre I'ARA et le potentiel hydrique de base (fig $5 \mathrm{a}$ ) montre que, pour un niveau de potentiel hydrique donné, I'ARA obtenue est plus élevée en présence de potassium.
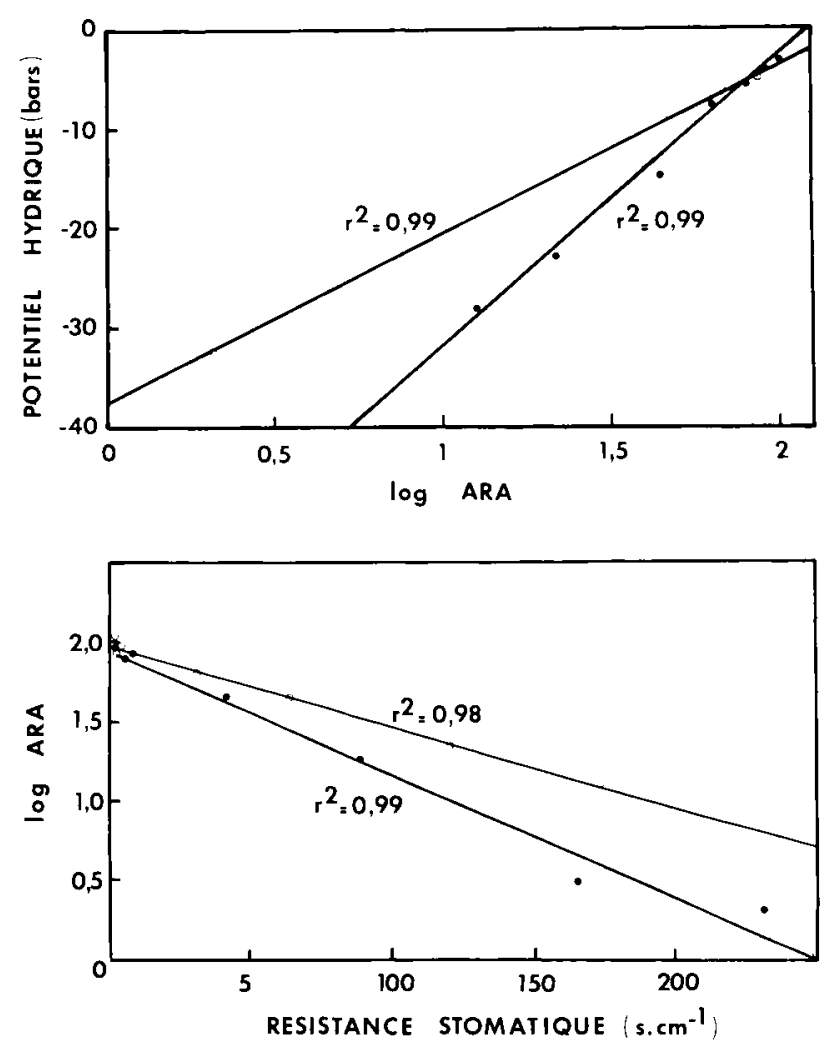

Fig. 5 a. Relation entre l'ARA et le potentiel hydrique de base. $\bigcirc \mathrm{K}_{0}, \mathrm{~K}_{1}$.

b. Relation entre l'ARA et la résistance stomatique. $O \mathrm{~K}_{0}$, - $\mathrm{K}_{1}$. 
La relation entre l'ARA et la résistance stomatique montre que l'ARA décroît avec l'augmentation de la résistance stomatique (fig $5 b$ ). Les courbes linéarisées ont des pentes significativement différentes indiquant un effet significatif du $\mathrm{K}$; pour un niveau de résistance stomatique donné, l'ARA observée est plus élevée en absence de $\mathrm{K}$.

Cette étude montre l'importance de l'alimentation potassique d'une culture de légumineuse fourragère susceptible de subir des conditions limitantes d'alimentation en eau.

Le trèfle blanc bien alimenté en $K$ conserve une activité fixatrice notable et accuse une moindre diminution de la production de matière sèche en conditions hydriques défavorables.

\section{RÉFÉRENCES}

Albrecht L, Benneth JM, Boote KJ (1984) Relationship of nitrogenase activity to plant water stress in field grown soybeans. Field Crops Res 8, 61-71

Aussenac G, Granier A (1978) Quelques résultats de cinétique journalière du potentiel de sève chez les arbres forestiers. Ann Sci For 35, 19-32

Barbarick KA (1985) Potassium fertilization of alfalfa grown on a soil high in potassium. Agron J 77, 442445

Barta AL (1982) Response of symbiotic N2 fixation and assimilate partitioning to $K$ supply in alfalfa. Crop Sci 22, 89-92

Brag $H$ (1972) The influence of potassium on the transpiration rate and stomatal opening in Triticum aestivum and Pisum sativum. Physiol Plant 26, 250257

Collins M, Land DJ (1985) Shoot removal and potassium fertilization effects on growth, nodulation and dinitrogen fixation of red clover and birdsfoot trefoil under greenhouse conditions. Field Crop Res 10, 251-256

Cooper RB, Blaser RE, Brown RH (1967) Potassium nutrition effects on net photosynthesis and morphology of alfalfa. Soil Sci Soc Am Proc 31, 231-235

Dhindsa RS, Beasley CA, Ting LP (1975) Osmoregulation in cotton fibre. Plant Physiol 56, 394-398

Duke SH, Collins M, Soberalske RM (1980) Effects of potassium fertilization on nitrogen fixation and nodule enzymes of nitrogen metabolism in alfalfa. Crop Sci 20, 213-219

Guckert A Laperrière C (1987) Effet du stress hydrique sur la fixation d'azote par le trèfle blanc (Trifolium repens L.). Les Coll. de I'INRA, 37, 245250

Humble GD, Raschke K (1971) Stomatal opening quantitatively related to potassium transport. Plant Physiol 48, 447-453

Koch B, Evans HJ (1966) Reduction of acetylene to ethylene by soybean root nodules. Plant Physiol, 47, 453-456

Laperrière $C$ (1984) Etude de la fixation de l'azote par le trèfle blanc. Aspects biologiques et agronomiques. Thèse INPL, ENSAIA Nancy, 99p

Mengel $K$ (1984) Le potassium en physiologie végétale. CR Acad Agric Fr 70, 1365-1376

Mengel K, Arneke WW (1982) Effect of potassium on the water potential, the pressure potential, the osmotic potential and cell elongation in leaves of Phaseolus vulgaris. Physiol Plant 54, 402-408

Obaton M, Miquel M, Robin P, Conejero C, Domenach AM, Bardin R (1982) Influence du déficit hydrique sur l'activité nitrate réductase et nitrogénase chez le soja (Glycine max. L. Merr. cv Hodgson). CR Acad Sci, série III, 294, 1 007-1 012

Peoples TR, Koch DW (1979) Role of potassium in carbon dioxide assimilation in Medicago sativa $\mathrm{L}$. Plant Physiol 63, 878-881

Raschke K (1975) Stomatal action. Annu Rev Plant Physiol 26, 309-340

Saxena NP (1985) Le rôle du potassium dans la résistance à la sécheresse. Revue de la potasse, section 16(5), 1-15

Schölander PF, Hammel HT, Bradstreet ED, Hemmingsen EA (1965) Sap pressure in vascular plants. Science 148, 339-346

Shamsun-Noor L, Robin C, Schontz D, Heulin T, Guckert A (1989) Effet d'un déficit hydrique sur le trèfle blanc (Trifolium repens L.). I. Importance du cultivar. Agronomie 9, 251-257

Sprent JI (1972) The effects of water stress on nitrogen-fixing root nodules. IV. Effects on whole plants of Vicia faba and Glycine max. New Phytol 71, 603611

Van Bavel CHM, Nakayama FS, Ehrler WL (1965) Measuring transpiration resistance of leaves. Plant Physiol 40, 535-540

Wahab SA (1984) Potassium nutrition and nitrogen fixation by nodulated legumes. Fert Res 8, 9-20

Zimmerman U (1978) Physics of turgor and osmoregulation. Annu Rev Plant Physiol 29, 141-148 